\title{
Improving Anti-Cancer Potentiality and Bioavailability of Gallic Acid by Designing Polymeric Nanocomposite Formulation
}

\author{
Hanaa H Ahmed ${ }^{1}$, Asmaa F Galal2*, Aziza B Shalby ${ }^{1}$, Ahmed A Abd-Rabou ${ }^{1}$, \\ Fathy M Mehaya ${ }^{3}$
}

\begin{abstract}
Objective: In this study, we investigated the in vivo antitumor activity and pharmacokinetic characteristics of encapsulated GA-NC (gallic acid nanocomposite) in normal and hepatocellular carcinoma (HCC)-induced rats. Methods: Rats were distributed into 4 groups; negative control, HCC, gallic acid (GA), and GA-NC. Serum levels of alpha-fetoprotein (AFP), endoglin (ENG), heat shock protein-70 (HSP-70), pro-caspase 3, lipocalin-2 (LCN-2) and $\beta$-cell leukemia/lymphoma 2 (Bcl-2) were assayed by ELISA. The pharmacokinetic parameters for GA or GA-NC were determined by means of non-compartmental approach based on the serum- concentration profiles of free GA and GA-NC after oral administration. Also, histological procedures were used for examination of liver tissue sections. Results: Anaplastic changes in liver tissues were observed in untreated HCC group, as well as a significant increase in the serum AFP level. In addition, significant elevation in the serum ENG level as an angiogenic marker and the serum levels of the apoptotic mediators; HSP-70, Bcl-2 and pro-caspase 3 beside significant amplification in the serum inflammatory modulator, LCN-2 were recorded. Treatment with free GA or GA-NC markedly recovered the anaplastic changes in the rat liver tissues. In addition, they restored serum levels of AFP, ENG, HSP-70, Bcl-2, pro-caspase-3, and LCN-2. Pharmacokinetic analysis revealed that GA-NC displayed a characteristic sustained release profile with 4-fold increase in bioavailability in normal and HCC-induced rats. Conclusions: The results of this study suggest that encapsulation of GA into PLGA-CS-PEG enhances its oral bioavailability and anti-cancer activity. GA-NC may be a new therapeutic candidate for the mitigation of hepatocarcinogenesis.
\end{abstract}

Keywords: Gallic acid- gallic acid nanocomposite- anti-cancer- pharmacokinetic- in vivo- bioavailability

Asian Pac J Cancer Prev, 19 (11), 3137-3146

\section{Introduction}

Globally, hepatocellular carcinoma (HCC) is considered as the third main cause of cancer-related mortality. In Egypt, HCC is the second recorded cancer type in men and the sixth type in women (Ziada et al., 2016). The Hepatitis C and B viruses (HCV and HBV), obesity, alcohol consumption, dietary iron overload, and dietary carcinogens; such as aflatoxins B1 and nitrosamines, are implicated in HCC etiology (Sadeeshkumar et al., 2016). Several treatment strategies are available for HCC like curative resection, radiofrequency ablation, liver transplantation, radioembolization and systemic targeted chemotherapy (Samarakoon et al., 2017). Chemotherapy causes survival of HCC patients with severe adverse events such as diarrhea, hand-foot skin disease, fatigue, anorexia and alopecia (Kumar et al., 2016). Besides, the development of multidrug resistance (MDR) to chemotherapy becomes an obstacle facing HCC therapy. Those serious side effects encourage searching for new anticancer agents for HCC with fewer adverse effects (Liang et al., 2010).

Nature is a wealthy source of diverse and complex compounds displaying different remarkable biological effects. Gallic acid, GA; 3, 4, 5-trihydroxy benzoic acid, is a potent natural phenolic compound present in various fruits, nuts, green tea and oak (Alves et al., 2016). This unique molecule exhibits a variety of biochemical and pharmacological properties like anti-inflammation (Hsiang et al., 2013; Yoon et al., 2013), antimutagenic (Liao et al., 2017), antitumor (Chen and Chang, 2012; Reddy et al., 2012; Ho et al., 2013), antioxidation (Kim et al., 2002), neuroprotective (Ban et al., 2008; Nabavi et al., 2012; Mansouri et al., 2013) and cardiovascular protective (Priscilla and Prince, 2009; Umadevi et al., 2012). Interestingly, GA has inhibitory effects on cancer cell growth at different molecular levels either in chemical-induced carcinogenic models (Verma et al., 2013; Aglan et al., 2017) or in vitro cancerous cell lines (Abd-Rabou and Ahmed, 2017). GA selectively 
inhibits cancer cell growth via the modulation of genes that encodes for cell cycle, metastasis, apoptosis and angiogenesis (Heidarian et al., 2016; Perazzoli et al., 2017). Its outstanding anticancer activity makes this compound an important therapeutic biomolecule with little or almost no toxicity upon normal cells (Chia et al., 2010). However, a major obstacle that limits the clinical use of GA is its low bioavailability. Oral administration of GA showed poor absorption and rapid metabolism and elimination (Ferruzzi et al., 2009; Bhattacharyya et al., 2013). Considering the rapid advancement in nanotechnology in the last few years, innovated strategies have been implemented to influence the disposition and efficiency of drugs through the use of nanocarriers. Nanocarrier-based delivery systems decrease systemic toxicity, maintain controlled and sustained release of drugs, and overlap drug resistance (Giodini et al., 2017). Biodegradable polymeric nanocarriers, such as polylactic-co-glycolic acid; PLGA), have emerged as a versatile carrier system for targeted delivery of anti-cancer drugs owing to its great biocompatibility and biodegradability. In addition, the good variability of chemical structures through chemical modification results in flexibility of physicochemical characteristics enabling its diverse drug delivery applications (Tang et al., 2010).

A recent work of our research team provided a clear evidence for the ability of GA encapsulation in PLGA-CS-PEG nanocomposite to improve its bioefficacy against hepatocellular carcinoma HepG2 and colorectal HCT 116 cells lines (Abd-Rabou and Ahmed, 2017). Thereby, these results motivated us to further investigate the efficacy of this new nanocomposite as a candidate therapy for HCC in vivo. As we hypothesized that PLGA-CS-PEG nanocomposite may provide higher oral bioavailability and better anti-cancer activity compared to free GA. Antitumor efficacy was tested in vivo through assessing tumor, angiogenic, apoptotic and inflammatory markers. In addition, histopathological description of liver tissue sections was carried out. Pharmacokinetic characteristics were evaluated after oral administration of either GA nanocomposite or free GA either in normal or HCC-induced rats.

\section{Materials and Methods}

\section{Chemicals and Drugs}

N-nitrosodiethylamine (NDEA), gallic acid, polyvinyl alcohol (PVA, MW 30.000), chitosan (MW 60.90 kDa, degree of deacetylation $85 \%$, derived from crab shells, in the form of fibrillar flakes), polyethylene glycol (2 kDa) were supplied from Sigma-Aldrich Chemicals Co. (St Louis, MO, USA). Poly (D, L-lactide-co-glycolide) (PLGA, copolymer ratio 50:50, inherent viscosity 0.41 ) was purchased from (Birmingham polymers, Inc., Birmingham, AL). Acetonitrile, methanol, and ethyl acetate HPLC grade were obtained from Fisher scientific (Pittsburgh, PA, USA). All the other chemicals were of high purity grade. Ultra pure water was used throughout and all solutions were passed through $0.22 \mu \mathrm{m}$ pore size filters.

\section{Animals}

Fifty adult female rats of Wistar strain weighing 170-200 g were acquired from the Animal House Colony of the National Research Centre, Giza, Egypt and housed in polypropylene cages in an environmentally controlled clean air room with a temperature of $25 \pm 1{ }^{\circ} \mathrm{C}$, an alternating $12 \mathrm{~h}$ light/12 h dark cycle, a relative humidity of $60 \pm 5 \%$ and ad libitum access to tap water and a standard rodent chow consisted of $10 \%$ casein, $4 \%$ salt mixture, $1 \%$ vitamin mixture, $10 \%$ corn oil, $5 \%$ cellulose and completed to $100 \mathrm{~g}$ with corn starch (Wadi El Kabda Co., Cairo, Egypt). The rats were allowed to adapt to the new environment for two weeks before the commencement of the experiment. The study protocol conformed to the ethical guidelines for animal experiment, which was approved by the Ethical Committee of the Medical Research of the National Research Centre, Giza, Egypt.

Preparation of GA-loaded PLGA-CS-PEG nanocomposites

For the preparation of PLGA-CS-PEG nanocomposite, PLGA nanocomposites were prepared by oil-in-water (O/W) single emulsion solvent evaporation method (Parveen and Sahoo, 2011) with slight modifications. Briefly, $100 \mathrm{mg}$ of PLGA polymer was dissolved in $3 \mathrm{ml}$ of organic solvent (chloroform) to form a primary emulsion which was further emulsified in an aqueous PVA solution ( $12 \mathrm{ml}, 2 \% \mathrm{w} / \mathrm{v})$, PEG ( $5 \% \mathrm{w} / \mathrm{w}$ and molecular weight $2 \mathrm{kD})$, and chitosan solution ( $12 \% \mathrm{w} / \mathrm{w}$ in $1 \%$ glacial acetic acid) to get PLGA-CS-PEG nanocomposite. Oil-in-water emulsion was formed using a microtip probe sonicator (VC 505, Vibracell Sonics, Newton, USA) set at 55W of energy output for 2 min over an ice bath. The emulsion was stirred overnight for the evaporation of the organic solvent. For therapeutic applications, GA-loaded PLGA-CS-PEG nanocomposite was prepared in a similar way as mentioned above, by adding a certain concentration of GA to the mixture prior emulsification.

\section{Characterization of the nanocomposite}

Calibration curve, dialysis and entrapment efficiency (EE) measurements

Calibration curve was generated for gallic acid using serial dilutions of each compound $(0,10,20,50,100 \mu \mathrm{M})$. All data were equipped and processed with the microplate reader (BMG Labtech, Germany). The compound entrapment efficiency (EE) was calculated from the ratio of the compound amount incorporated into the nanocomposite to the total added compound amount. The prepared nano-structures were enrolled to dialysis tubing technique for eliminating the impurities and the free non-conjugated compounds suspended in the solution by eluting it through regenerated cellulose (Amicon 10,000 MWCO ultra filter, Millipore, USA).

\section{Particle size and zeta potential analyses}

Particle size and zeta potential of the nanocomposites were determined by photon correlation spectroscopy (PCS) using a Zeta Sizer (Nano ZS, Malvern Instruments, UK). All the samples were maintained at a constant temperature of $25.0^{\circ} \mathrm{C}$. Moreover, all experiments and each batch were 
analyzed in triplicates.

\section{Experimental setting}

The animals were randomly distributed into 4 groups, with 10 rats in each group. Group (1): set as healthy control group [negative control] received orally $0.9 \%$ normal saline daily during the experimental period. Group (2): served as hepatocellular carcinoma group [HCC group] in which the rats were orally administered with $\mathrm{N}$-nitrosodiethylamine (NDEA) (dissolved in $0.9 \%$ normal saline), in a dose of $20 \mathrm{mg} / \mathrm{kg} \mathrm{b}$. wt. five times weekly for four weeks followed by $10 \mathrm{mg} / \mathrm{kg}$ b.wt five times a week for another one week according to the method of Darwish et al. (Darwish and El-Boghdady, 2011). Group (3): gallic acid-treated group [HCC + GA] in which $\mathrm{HCC}$ afflicted rats were treated orally with free form of gallic acid in a dose of $50 \mathrm{mg} / \mathrm{kg}$ b.wt daily for 5 weeks (Karimov et al., 2003). Group (4): Gallic acid PLGA-CS-PEG -treated group [HCC + GA-NC] in which $\mathrm{HCC}$ afflicted rats were treated orally with gallic acid nanocomposite in a dose of $50 \mathrm{mg} / \mathrm{kg} \mathrm{b}$.wt daily for 5 weeks.

After the last treatment, the animals were fasted overnight and the blood samples were withdrawn, under diethyl ether anesthesia, from the retro-orbital venous plexus in a dry clean centrifuge tubes and allowed to coagulate for 45 minutes at room temperature to obtain sera. Serum samples were separated by centrifugation at $1,800 \times \mathrm{g}$ for 15 minutes at $4^{\circ} \mathrm{C}$ using cooling centrifuge and stored at $-20^{\circ} \mathrm{C}$ pending further analysis. After collection of the blood samples, the animals were sacrificed by cervical dislocation and the whole liver of each rat was rapidly and carefully dissected then, fixed in formalin saline $(10 \%)$ for histopathological investigation.

\section{Biochemical determinations}

Serum levels of AFP and ENG were estimated using rat ELISA kits purchased from Elabscience Biotechnology Co., Ltd (P.R.C.), according to the manufacturer's instructions provided with the assay kits. Serum levels of HSP-70, pro-caspase 3 and LCN-2 were quantified using rat ELISA kits purchased from WKEA MED Supplies Corp Co., Ltd (China) according to the manufacturer's manual provided with assay the kits. Serum level of Bcl-2 was determined by rat ELISA kit purchased from Glory Science Co (USA), according to the manufacturer's instructions provided with assay the kit.

\section{Histological examination}

The fixed liver specimens were trimmed, washed and dehydrated in ascending grades of alcohol. Tissue specimens were then cleared in xylene, embedded in molten paraffin, sectioned at 4-6 microns thickness using rotary microtone, stained with Hematoxylen and Eosin ( $\mathrm{H}$ and $\mathrm{E}$ stain) and examined under the light microscope (Carleton, 1976).

\section{Pharmacokinetic study}

Rats were randomly allocated into 4 groups $(n=6)$ : group I: normal rats received single dose of free GA (50 mg/kg b.wt, p.o.), group II: HCC-induced rats received single dose of free $\mathrm{GA}(50 \mathrm{mg} / \mathrm{kg}$ b.wt, p.o.), group III: normal rats received single dose of gallic acid nanocomposite (50 mg/kg b.wt, p.o.), and group IV: HCC-induced rats received single dose of gallic acid nanocomposite (50 mg/kg b.wt, p.o.). Rats were fasted for $12 \mathrm{~h}$ with free access to water prior to experiments. Blood samples $(0.5 \mathrm{ml})$ were collected at $0.083,0.15,0.30,0.5$, 1, 2, 3, 4, 6 and 24 hours following drug administration. Blood samples were immediately centrifuged at $1800 \mathrm{xg}$ for $10 \mathrm{~min}$; sera were separated and stored at $-20^{\circ} \mathrm{C}$ until analysis.

\section{Sample preparation}

A liquid-liquid extraction (LLE) method was applied to extract gallic acid from serum samples according to Sun et al. (2014) with some modifications. Briefly, serum sample $(250 \mu \mathrm{l})$ was spiked with $25 \mu \mathrm{l}$ of pyrogallol $(1 \mathrm{mg} / \mathrm{ml})$, $25 \mu \mathrm{l}$ of $\mathrm{HCl}$ and $1 \mathrm{~mL} 0.9 \% \mathrm{NaCl}$ (in dist. water), vortex mixed for $30 \mathrm{~s}$ and then incubated for $10 \mathrm{~min}$. in ultrasonic bath. After these procedures, the mixture was extracted with ethyl acetate by shaking on a vortex mixer for $3 \mathrm{~min}$ at room temperature. After centrifugation at 3,000 $\mathrm{xg}$ for $5 \mathrm{~min}$, the supernatant was transferred to a clean tube and evaporated to dryness under a gentle stream of nitrogen at $40^{\circ} \mathrm{C}$. The residue was reconstituted in $100 \mu \mathrm{l}$ methanol, filtered using a $0.45 \mu \mathrm{m}$ nylon membrane filter and $75 \mu \mathrm{l}$ was injected into the HPLC system.

\section{Instrumentation and chromatographic conditions}

The chromatographic analysis was performed on Agilent HPLC system (HP 1,100 series), equipped with auto-sampler, quaternary pump, on-line degasser and diode array detector, controlled with chemstation software (Hewlett Packard, Waldbronn, Germany). The analytical column was Agilent Zorbax XDB C18 column (150 x $4.6 \mathrm{~mm}$ I.D., $5 \mu \mathrm{m}$, Germany) fitted with a guard cartridge packed with similar stationary phase. The separation of GA was performed as gradient separation with acetonitrile (solvent A) and acetic acid 2\% (solvent B) with a flow rate of $1.0 \mathrm{ml} / \mathrm{min}$. The detection wavelength was $280 \mathrm{~nm}$. GA concentrations in the samples were calculated by comparing the corresponding peak areas of the sample to GA standard solutions.

\section{Pharmacokinetics and statistical analysis}

The pharmacokinetic parameters for GA or GA-NC were determined by means of non-compartmental approach based on the serum- concentration profiles of free GA and GA-NC after oral administration. The area under the curve (AUC0-t) and the area under the first-moment curve (AUMC0- $t$ ), where $t$ is the last sampling time, were calculated by the linear trapezoidal rule method. The area under the curve extrapolated to infinity $(\mathrm{AUC} 0-\infty)$ was obtained by the equation $\mathrm{AUC} 0-\infty=\mathrm{AUC} 0-\mathrm{t}+\mathrm{Cp} / \mathrm{ke}$, where $\mathrm{Cp}$ is the last measurable concentration and ke is the elimination rate constant. The AUMC0- $\infty$ was calculated as $\mathrm{AUMC} 0-\mathrm{t}+(\mathrm{Cp} \times \mathrm{t} / \mathrm{ke})+(\mathrm{Cp} / \mathrm{ke} 2)$. Mean residence

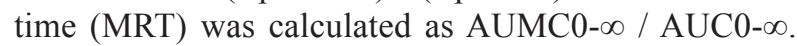
The slope of linear regression of the log transformed concentration versus time in the terminal phase was used to obtain ke. All data analysis was performed with Kinetica 5 
(Build 5.0.11) software (Thermo Fisher Scientific, USA). Data were expressed as mean \pm standard errors (SE). Group differences were determined by analysis of variance using Tukey's post-hoc test (Graph Pad Prism 5.01 software).

\section{Results}

\section{Characterization of GA nanocomposites}

GA-load PLGA-CS-PEG nanocomposites were synthesized and characterized as previously mentioned in our recent publication (Abd-Rabou and Ahmed, 2017). For measuring the entrapment efficiency (EE) of GA in the nanocomposite, calibration curve after dialysis tubing was carried out. We found that the EE was approximately $80 \%$ of the total concentration used in the nanocomposite. Dynamic Light Scattering (DLS) analysis showed that their Z-average diameters were around $295 \pm 5.5 \mathrm{~nm}$. The polydispersity index (PDI) was 0.1 . There was a consistent pattern of positive charge (zeta potential) on the surface of gallic acid-encapsulated PLGA-CS-PEG nanocomposite as shown in Abd-Rabou and Ahmed (2017).

\section{In vivo antitumor testing}

In this study, rats afflicted with HCC experienced significant amplification $(\mathrm{P}<0.05)$ in serum AFP (54.7\%), ENG (297.4\%) and HSP-70 (107.8\%) levels in comparison with the negative control counterparts. In contrast, treatment with $\mathrm{GA}$ or $\mathrm{GA}-\mathrm{NC}$ in HCC group yielded significant decline $(\mathrm{P}<0.05)$

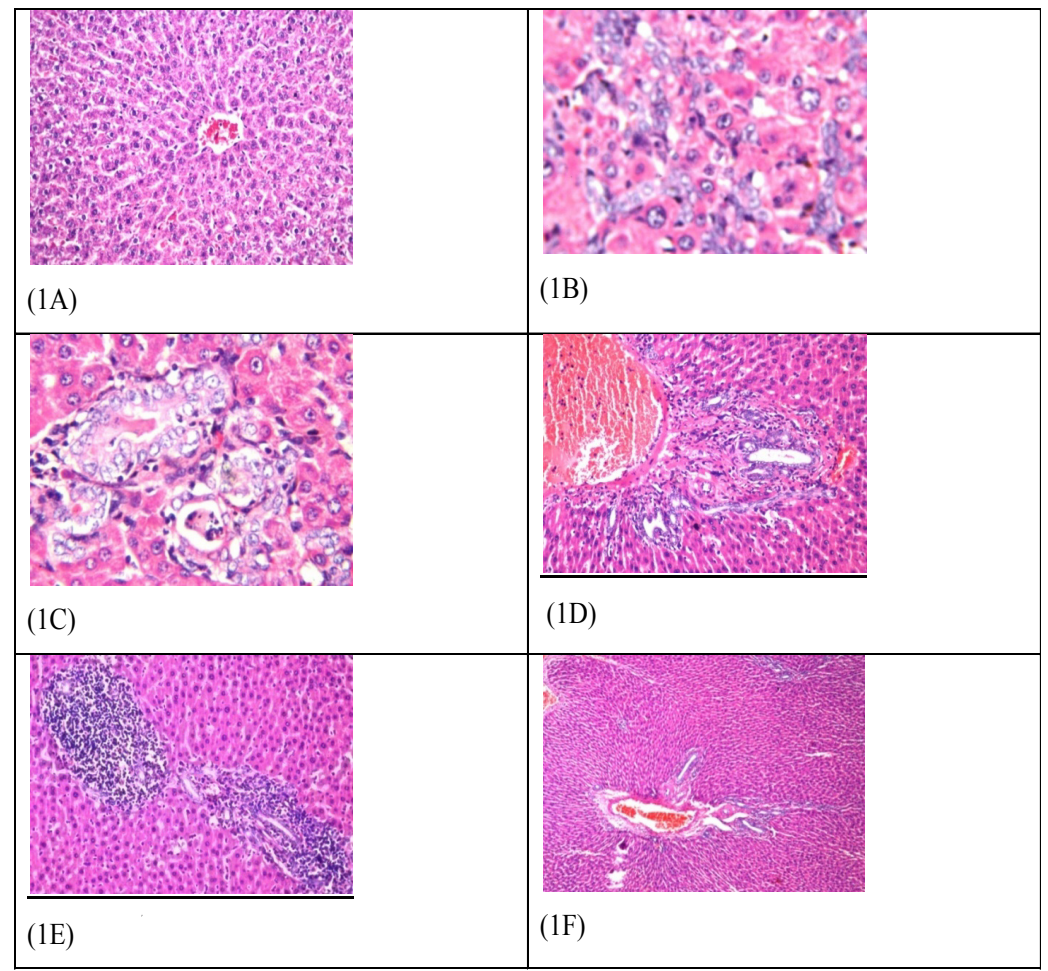

Figure 1. Photomicrograph of Liver Section of (1A) Negative Control Group Showing Normal Histological Structure of Central Vein (cv) and Surrounding Hepatocytes in the Parenchyma. (H and E x 10), (1B) HCC group showing karyomegalic nuclei of the anaplastic hepatocytes with trabecular anaplastic arrangement and glandular pattern $(1 \mathrm{C}) .(\mathrm{H}$ and $\mathrm{E} x$ 40), (1D) HCC + GA shows congestion in the portal vein, multiple newly formed bile ducts and inflammatory cells infiltration in between at the portal area as well as focal aggregations of mononuclear leucocytes inflammatory cells were detected in the hepatic parenchyma (1E) ( $\mathrm{H}$ and $\mathrm{E} x \mathrm{40}),(1 \mathrm{~F}) \mathrm{HCC}+\mathrm{GA}-\mathrm{NC}$ showing congestion in the portal vein, newly formed bile ducts and inflammatory cells infiltration in between (HandE x 40).

Table 1. Influence of Treatment with Gallic Acid (GA) and Gallic Acid Nanocomposite (GA-NC) on Serum Alpha-Fetoprotein (AFP), Endoglin (ENG) and Heat Shock Protein-70 (HSP-70) Levels in Hepatocellular Carcinoma (HCC) Afflicted Rats

\begin{tabular}{lccc}
\hline Groups & AFP ng/ml & ENG ng/ml & HSP-70 ng/ L \\
\hline Negative control & $38.06 \pm 0.50$ & $3.11 \pm 0.17$ & $31.88 \pm 1.341$ \\
HCC & $58.88 \pm 1.863^{\mathrm{a}}$ & $12.36 \pm 0.82^{\mathrm{a}}$ & $66.24 \pm 1.383^{\mathrm{a}}$ \\
& $(54.7 \%)$ & $(297.4 \%)$ & $(107.8 \%)$ \\
$\mathrm{HCC}+$ GA & $41.19 \pm 0.83^{\mathrm{b}}$ & $4.16 \pm 0.14^{\mathrm{b}}$ & $43.56 \pm 0.8426^{\mathrm{b}}$ \\
& $(-30.04 \%)$ & $(-66.3 \%)$ & $(-34.2 \%)$ \\
HCC + GA-NC & $39.10 \pm 0.57^{\mathrm{b}}$ & $3.50 \pm 0.14^{\mathrm{b}}$ & $39.11 \pm 1.141^{\mathrm{b}}$ \\
& $(-33.6 \%)$ & $(-71.7 \%)$ & $(-40.95 \%)$ \\
\hline
\end{tabular}

${ }^{\text {a }}$ Significant difference versus the negative control group; ${ }^{\mathrm{b}}$ Significant difference versus HCC group 
Table 2. Influence of Treatment with Gallic Acid (GA) and Gallic Acid Nanocomposite (GA-NC) on Serum $\beta$-cell Leukemia/lymphoma 2 (Bcl-2), Pro-caspase 3 and Lipocalin-2 (LCN-2) Levels in Hepatocellular Carcinoma (HCC) Afflicted Rats

\begin{tabular}{lccc}
\hline Groups & Bcl-2 ng/ml & Pro-caspase 3 ng/ml & LCN-2 ng/ L \\
\hline Negative control & $5.66 \pm 0.28$ & $0.75 \pm 0.0234$ & $236.1 \pm 4.65$ \\
HCC & $20.04 \pm 0.377^{\mathrm{a}}$ & $3.87 \pm 0.124^{\mathrm{a}}$ & $384.3 \pm 3.42^{\mathrm{a}}$ \\
& $(254 \%)$ & $(416 \%)$ & $(62.7 \%)$ \\
HCC + GA & $13.44 \pm 0.37^{\mathrm{b}}$ & $1.53 \pm 0.044^{\mathrm{b}}$ & $250.3 \pm 1.39^{\mathrm{b}}$ \\
& $(-32.9 \%)$ & $(-60.5 \%)$ & $(-34.8 \%)$ \\
HCC + GA-NC & $11.78 \pm 0.24^{\mathrm{b}}$ & $1.37 \pm 0.052^{\mathrm{b}}$ & $247.9 \pm 2.28^{\mathrm{b}}$ \\
& $(-41.2 \%)$ & $(-64.6 \%)$ & $(-35.5 \%)$ \\
\hline
\end{tabular}

aignificant difference versus negative control group, ${ }^{\mathrm{b}}$ Significant difference versus HCC group

Table 3. Pharmacokinetic Parameters of Gallic Acid (GA) and Gallic acid Nanocomposite (GA-NC) in Normal and Hepatocellular Carcinoma (HCC)-induced Rats

\begin{tabular}{|c|c|c|c|c|}
\hline & $\begin{array}{l}\mathrm{GA}(50 \mathrm{mg} / \mathrm{kg}) \text { in } \\
\text { normal rats }\end{array}$ & $\begin{array}{l}\mathrm{GA}(50 \mathrm{mg} / \mathrm{kg}) \text { in } \\
\text { HCC-afflicted rats }\end{array}$ & $\begin{array}{c}\text { GA-NC }(50 \mathrm{mg} / \mathrm{kg}) \text { in } \\
\text { normal rats }\end{array}$ & $\begin{array}{l}\text { GA-NC }(50 \mathrm{mg} / \mathrm{kg}) \text { in } \\
\text { HCC-afflicted rats }\end{array}$ \\
\hline $\mathrm{C}_{\max }(\mu \mathrm{g} / \mathrm{ml})$ & $1.57 \pm 0.108$ & $0.923^{\mathrm{a}} \pm 0.33$ & $2.45^{\mathrm{a}} \pm 0.07$ & $2.62^{\mathrm{a}} \pm 0.18$ \\
\hline $\mathrm{T}_{\max }(\mathrm{h})$ & $0.46 \pm 0.085$ & $0.87^{\mathrm{a}} \pm 0.25$ & $0.625 \pm 0.125$ & $2.00^{\mathrm{a}, \mathrm{b}} \pm 0.0$ \\
\hline $\mathrm{T}_{1 / 2}(\mathrm{~h})$ & $0.62 \pm 0.09$ & $1.14 \stackrel{\mathrm{a}}{ \pm} 0.32$ & $1.22^{\mathrm{a}} \pm 0.15$ & $2.09^{\mathrm{a}, \mathrm{b}} \pm 0.02$ \\
\hline AUClast ( $\left.\mu \mathrm{g} \cdot \mathrm{ml} \cdot \mathrm{h}^{-1}\right)$ & $1.641 \pm 0.045$ & $1.34 \pm 0.194$ & $6.25^{\mathrm{a}} \pm 0.188$ & $7.3^{\mathrm{a}, \mathrm{b}} \pm 0.8$ \\
\hline AUC $\left(\mu \mathrm{g} \cdot \mathrm{ml} \cdot \mathrm{h}^{-1}\right)$ & $1.89 \pm 0.13$ & $1.67 \pm 0.04$ & $6.52^{\mathrm{a}} \pm 0.31$ & $7.52^{\mathrm{a}, \mathrm{b}} \pm 0.8$ \\
\hline $\mathrm{AUMC}_{\text {last }}\left(\mu \mathrm{g} \cdot \mathrm{ml} \cdot \mathrm{h}^{-1}\right)$ & $1.3 \pm 0.13$ & $1.55 \pm 0.014$ & $11.29^{\mathrm{a}} \pm 1.66$ & $13.56^{\mathrm{a}, \mathrm{b}} \pm 1.6$ \\
\hline AUMC $\left(\mu \mathrm{g} \cdot \mathrm{ml} \cdot \mathrm{h}^{-1}\right)$ & $2.03 \pm 0.44$ & $1.55 \pm 0.014$ & $13.36^{\mathrm{a}} \pm 2.7$ & $14.65^{\mathrm{a}} \pm 1.6$ \\
\hline MRT (h) & $1.063 \pm 0.155$ & $1.43 \pm 0.34$ & $2.037^{\mathrm{a}} \pm 0.322$ & $1.96^{\mathrm{a}} \pm 0.03$ \\
\hline
\end{tabular}

${ }^{\mathrm{a}}$ significantly different from GA in normal rats, ${ }^{\mathrm{b}}$ significantly different from GA-NC in normal rats, ${ }^{\mathrm{c}}$ significantly different from GA in HCC-afflicted rats

in serum AFP $(-30.04 \%,-33.6 \%$ respectively), ENG $(-66.3 \%,-71.7 \%$ respectively) and HSP-70 $(-34.2 \%,-40.95 \%$ respectively) levels versus the HCC group (Table 1).

Serum Bcl-2, pro-caspase 3 and LCN-2 levels revealed significant rise in HCC group in comparison with the negative control group. Conversely, treatment of HCC afflicted rats with GA or GA-NC exhibited significant reduction $(\mathrm{P}<0.05)$ in serum $\mathrm{Bcl}-2(-32.9 \%,-41.2 \%$, respectively), pro-caspase $3(-60.5 \%,-64.6 \%$, respectively) and LCN-2 (-34.8\%, $-35.5 \%$, respectively) levels with

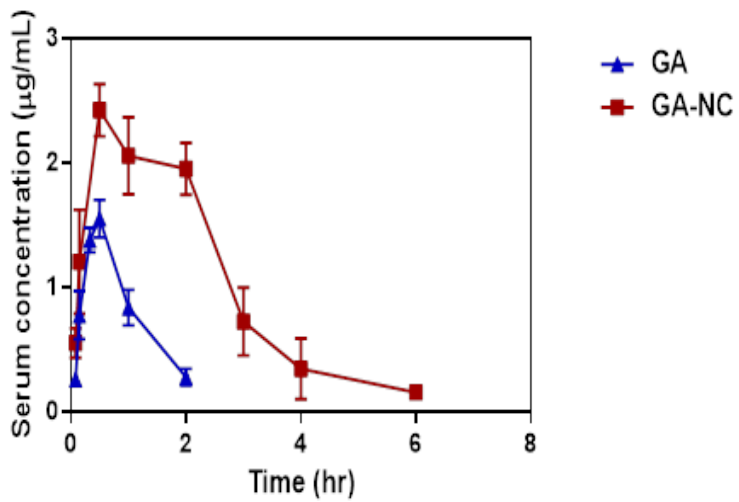

Figure 2. Time Course of Gallic Acid (GA) in the Serum of Normal Rats after Oral Administration of ( $\_$) GA, ( - ) Gallic Acid Nanocomposite (GA-NC). GA was measured in serum after oral administration by HPLC. Each data point represents the mean \pm S.D. of 6 rats. respect to the HCC counterparts (Table 2).

\section{Histopathological findings}

Photomicrograph of liver tissue section of rat in the negative control group revealed normal histological structure of the central vein and encompassing hepatocytes in the hepatic parenchyma as recorded in Figure (1A). Meanwhile, photomicrograph of liver tissue section of rats in HCC group indicated karyomegalic nuclei of the anaplastic hepatocytes with trabecular anaplastic arrangement (Figure 1B) and glandular pattern (Figure 1C).

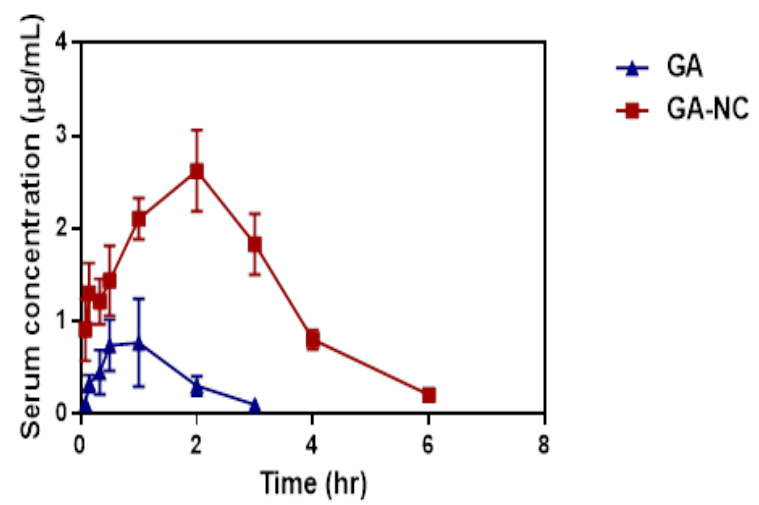

Figure 3.Time Course of Gallic Acid (GA) in the Serum of HCC-induced Rats after Oral Administration of ( $\_$) GA, ( - - ) Gallic Acid Nanocomposite (GA-NC). GA was measured in serum after oral administration by HPLC. Each data point represents the mean \pm S.D. of 6 rats. 
Photomicrograph of liver tissue section of rat afflicted with HCC and treated with GA represented congestion in the portal vein, multiple newly formed bile ducts and inflammatory cells infiltration in between at the portal area (Figure 1D). Also, focal aggregations of mononuclear leucocytes inflammatory cells were detected in the hepatic parenchyma (Figure 1E). Photomicrograph of liver tissue section of rat afflicted with $\mathrm{HCC}$ and treated with GA-NC revealed congestion in the portal vein, newly formed bile ducts and inflammatory cells infiltration in between (Figure 1F).

\section{Pharmacokinetic study}

The developed LLE method was successfully applied to compare the pharmacokinetic profiles of free GA and GA-NC in normal and $\mathrm{HCC}$-induced rats.

\section{Free Gallic acid}

The mean concentration-time curve of GA after administration of free GA (50 mg/kg b.wt.), in normal and HCC-induced rats, respectively, is illustrated in Figures 2 and 3. The pharmacokinetic parameters are presented in Table (3). In normal rats, serum concentration-time profile of free GA was characterized by rapid absorption and disposition. Serum concentrations increased rapidly to reach $\mathrm{C}_{\text {max }}$ value of $1.587 \mu \mathrm{g} / \mathrm{mL}$ at $\mathrm{T}_{\text {max }}$ value of $0.46 \mathrm{~h}$ and declined rapidly, showing duration of action of approximately $1 \mathrm{hr}$. Elimination half-life was $0.62 \mathrm{hr}$ and MRT was $1.06 \mathrm{hr}$.

Serum concentration-time profile of gallic acid showed different pattern in HCC-induced rats. Liver cancer had a substantial effect on pharmacokinetic behavior of GA compared to matched healthy rats. The $\mathrm{C}_{\max }$ decreased, while $\mathrm{T}_{\max }$, MRT and $\mathrm{t} 1 / 2$ were doubled, indicating that the absorption process became slow. Moreover, our results

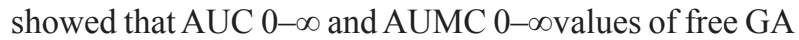
in the $\mathrm{HCC}$ afflicted rats were slightly decreased compared to those of normal control counterparts indicating a decrease in fraction absorbed.

\section{Gallic acid nanocomposite}

As shown in Table 3, after oral administration of GA-NC (50 mg/kg b.wt.), the maximum serum drug concentrations of approximately $2.45 \pm 0.07 \mu \mathrm{g} / \mathrm{mL}$ and $2.65 \pm 0.18 \mu \mathrm{g} / \mathrm{mL}$ were observed at $0.625 \mathrm{~h}$ and $2.00 \mathrm{~h}$ in normal and $\mathrm{HCC}$-induced rats, respectively. After $\mathrm{C}_{\max }$ was attained, serum concentrations decreased gradually, resulting in a decrease of about $20 \%$ over $2 \mathrm{hr}$ following $\mathrm{T}_{\max }$. This peak was followed by a gradual drop between 2 and $6 \mathrm{hr}$.

We observed that peak serum concentration of GA-NC was about 4-fold higher than that of free GA. The elimination half-time (t1/2) of GA-NC was prolonged about 2-fold than that of free GA $(1.22 \pm 0.15 \mathrm{hr}$ for GA-NC and $0.62 \pm 0.09,1.4 \pm 0.32 \mathrm{hr}$ for free GA in normal and $\mathrm{HCC}$-induced rats, respectively) and mean residence time (MRT0- t) was doubled (2.037 vs 1.063 and 1.96 vs 1.43 in normal and HCC-induced rats, respectively ) which indicated that free GA was cleared more slowly from rats serum for GA-NC than free GA. Moreover, the increase of AUC $(0-\infty)$ from $1.89 \pm 0.13 \mu \mathrm{g} \cdot \mathrm{mL} . \mathrm{hr}^{-1}$ for
GA to $6.52 \pm 0.31 \mu \mathrm{g} . \mathrm{mL} \cdot \mathrm{hr}^{-1}$ for GA-NC (4-fold) indicated that GA-NC could provide higher bioavailability than free GA.

\section{Discussion}

The present study proposed GA encapsulated in PLGA-CS-PEG as a new GA-based therapy for HCC. Oral administration of either GA or GA-NC modulated the levels of the tested biochemical parameters and mitigated HCC induced by NDEA in the experimental animals. In addition, the present study demonstrated that GA-NC was superior to free GA in terms of bioavailability and controlled release behaviour.

\section{In vivo Antitumor Activity}

Alpha-fetoprotein (AFP) is a relevant tumor marker that can be generated by hepatic tumors. It has been reported that exposure of rats to bearing HCC displayed an elevation in circulating AFP levels (Sadeeshkumar et al., 2016). Here, GA and GA-NC administration in $\mathrm{HCC}$-afflicted rats significantly decreased AFP serum level (Jagan et al., 2008). This comes in line with the study of Sur et al., (2016) who mentioned that GA, as a polyphenol, caused a remarkable reduction in AFP and CD44 expressions leading to the proliferation of hepatocyte progenitor and stem cells, respectively, in restriction of hepatic carcinogenesis.

Endoglin (ENG) is a key element for angiogenesis/ vascular development as it expressed at low levels in resting endothelial cells while its expression promptly increases in active vascular endothelial cells with cancer angiogenesis (Lebrin et al., 2005). The ENG gene, which is $40 \mathrm{~kb}$ in length, is located on the long (q) arm of chromosome 9 at position $9 \mathrm{q} 34$. Its protein is a $180 \mathrm{kD}$ a homo dimeric transmembrane glycoprotein that forms a complex with growth factors and different proteins included in vascular angiogenesis. Lebrin et al., (2005) reported that ENG expression alterations in cancer cells may contribute to the deregulation of TGF- $\beta$ (dependent and independent) signaling pathways and tumor growth. ENG is known to upregulate TGFß1 phosphorylation with the subsequent increase of Smad 2 protein phosphorylation. This process can clarify the role of these factors as integrators of various signals to modulate the gene transcription. This explains the role of carcinogenesis associated with the upregulation of ENG and TGF- $\beta 1$ genes expression in hepatocarcinogenesis and increased risk of HCC in individuals with cirrhosis (Teama et al., 2016). In the current study, the depressive effect of GA and GA-NC on serum ENG level in HCC-afflicted rats could be due to its suppression effect on phosphorylation of Smad proteins (Kee et al., 2016). Of note, a positive correlation has been found between serum levels of ENG and AFP in HCC patients (Mohamed et al., 2015).

A highly conserved heat shock proteins (HSPs) have vital functions that mediate cell survival. HSP70 has a special role in apoptotic signal transduction. It has been reported that HSP70 has contributed in many apoptotic signaling pathways, like attenuating cytosolic calcium and stabilizing lysosomes, suppressing stress kinases activities, 
inhibiting Bid or Bax translocation into mitochondria and suppressing the recruitment of procaspase- 9 to the Apaf-1 apoptosome (Wang et al., 2016). In HCC, HSP70 gene was over-expressed and it is one of the most upregulated genes in early hepatocellular carcinoma compared with non-neoplastic liver (Nguyen et al., 2016). GA and GA-NC administration in $\mathrm{HCC}$-induced rats in the current study significantly blunted serum HSP70 level. This could be explained by the ability of GA to attenuate the expression levels of heat shock protein (HSP) gene (Sobeh et al., 2016).

Anti-cancer agents are often able to induce apoptosis, thus eliminating cells that harbor genetic damage or exhibit inappropriate division. During apoptosis, cell mortality occurs in a well-controlled and regulated manner. Two major pathways (extrinsic and intrinsic) are involved in the regulation of apoptosis. Both these pathways converge to a common one in a cascade of proteases named "caspases," a family of enzymes that act as death effector molecules in various forms of cell death (Sadeeshkumar et al., 2016). The Caspase 3 activation pathway is an important downstream executioner in apoptosis (Rejiya et al., 2009). Moreover, Bcl-2 family members have been augmented to be other primary mediators of apoptosis in the mitochondrial-mediated pathway. Bcl-2 is an anti-apoptotic member, while Bax is a pro-apoptotic (tumor suppressor) member, which increases the mitochondrial permeability and accelerate apoptotic cell death (Mard et al., 2015; Sun et al., 2016). The output of the current study revealed that GA and GA-NC therapy could significantly decrease serum levels of Bcl-2 and pro-caspase-3. GA is able to activate apoptosis via mitochondrial pathway, and may have the potential to be a denovo therapeutic compound for use in the treatment of HCC (Sun et al., 2016).

Lipocalin 2 (LCN-2), a secreted protein, is a member of the Lipocalins family, a group of transporters of small lipophilic molecules such as steroids, lipopolysaccharides, and fatty acids in circulation. It has been suggested that there is an elevated level of circulated LCN-2 in HCC patients owing to LCN-2 over-expression in inflammatory cells, macrophage cell lines, epithelial cells, and liver cells (Abd El Moety et al., 2013; Asimakopoulou et al., 2016). LCN-2 level has been found to be elevated in HCC tissues and the higher of both LCN-2 and LCN-2R expression correlated with shorter overall survival in $\mathrm{HCC}$ patients (Asimakopoulou et al., 2016). The present work revealed that GA and GA-NC administration decreased the LCN-2 level in HCC-afflicted rats. This finding is greatly supported by the study of Chen et al., (2013) who reported that GA has a potential effect on the activation of c-Jun NH2-terminal kinase (JNK) which inhibited by elevation of serum LCN-2 level under the influence of polyphenol agents (Borkham-Kamphorst et al., 2013).

Our outputs are confirmed with histological investigation of hepatic tissue. NDEA, a N-nitroso compounds (NOC), is a mutagenic, teratogenic, and carcinogenic activities. So we can use it as a chemically induced HCC model (Santos et al., 2017). The present work presented that the therapeutic effect GA and GA-NC against HCC. Sun et al. (2016) explained the positive effect of GA on the liver tissue of HCC-afflicted rats due to its antiproliferative and apoptotic effect.

\section{In vivo Pharmacokinetic Study}

The pharmacokinetics analysis revealed that the newly developed GA-NC provided higher $\mathrm{C}_{\max }$ compared to free GA. The prolongation of half life, enhanced residence time and decreased clearance indicated that GA-NC posses better absorption, metabolism and elimination than free GA while providing an extended drug acting time. In order to be useful as anticancer treatment, a more sustained residence of GA is desirable. In this context, bioavailability, assessed as area under the curve

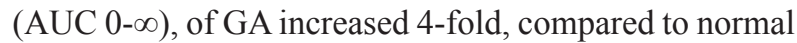
control pointing to the enhanced oral bioavailability of GA-NC relative to free GA. The prolongation of the mean residence time (MRT) is further confirming the retention in the systemic circulation and hence, sustained release pattern. It is clear that GA-NC formulation provides better pharmacokinetic properties than free GA. The system of GA-NC may possess better solubility and potential antitumor efficacy. Encapsulation of GA into nanocarriers offers a prominent increase in oral absorption and bioavailability, allows a controlled release in addition to protection against premature degradation through mucoadhesion.

The current findings implied that NDEA-induced liver cancer resulted in a decrease in absorption rate and absorption amount, decrease in system clearance, and increase in residence of GA, which confirmed that the study of the pharmacokinetic profiles of drugs in pathological state is more meaningful than in normal state. Therefore, it is valuable to provide the pharmacokinetic characteristic of GA in HCC-afflicted rats for better prediction of the clinical pharmacokinetic behaviors and guidance of the dosage regimen adjustment in clinical application. HCC is associated with alterations of the liver structure, distortion of metabolic functions and destruction of cell membrane structure, leading to a decrease in metabolizing enzymes capacity, and a reduction of hepatic blood flow. Accordingly, liver cancer might exert a complicated influence on drug absorption, disposition and clearance. The decrease in absorption rate and absorption amount observed in our study could be attributed to possible alteration in drug absorption and gut motility, probably as a result of abnormal concentrations of gut hormones such as motilin. Hence, the result is a delay in gastric emptying and orocaecal transit, reducing the rate and extent of absorption (Donelli et al., 1998). Furthermore, intestinal absorption of GA is known to be dependent on active intestinal transporters including the monocarboxylic acid transporter which can be affected by liver cancer. Modulation of expression and/ or activity would likely impact absorptive efficiency or bioavailability. These transporters are primarily responsible for transferring polyphenols from the intestinal lumen into the enterocyte to be partially metabolized, secreted into circulation or effluxed back to the intestinal lumen by inducible multidrug resistance transport proteins including P-glycoprotein and/or the Multi Drug Resistance Protein-2 (Vaidyanathan and Walle, 2001; Vaidyanathan 
and Walle, 2003; Konishi et al., 2004; Konishi et al., 2006).

Previous studies have unveiled that major biotransformation pathways of GA include glucuronidation, sulfation, hydrolization, and methyl conjugation, and three main metabolites have been identified. Among them, glucuronidation plays a critical role in the elimination of GA (Shahrzad and Bitsch, 1998; Feng, 2006). Depending on type of liver lesion, drug metabolism can be affected. Biliary excretion of drugs and oxidative drug metabolism has been shown to be impaired in subjects with hepatic cirrhosis whereas conjugation of drugs often remains unaffected (Pirmohamed, 2015). Cholestatic diseases are more likely to affect drug transporter proteins (phase III pathways), whereas phase I metabolism is relatively spared. In contrast, acute hepatic inflammation is more likely to down-regulate CYP enzyme via a nitric oxide dependent pathway (Gonthier et al., 2003).

In conclusion, the present work emphasized the pivotal role of gallic acid as a promising rival of hepatocellular carcinoma. The newly developed nanocomposite offers an attractive possibility of improving encapsulation efficiency and oral bioavailability. The current findings are encouraging and offer an attractive opportunity for harnessing maximum benefits out of a multitalented anticancer agent in a promising formulation able to sustain the drug release and decrease the dosage burden.

\section{Conflict of interest}

The author(s) disclosed receipt of the following financial support for the research, authorship, and/or publication of this article: This work was supported and funded by research project grant (ID 10010106) from National Research Centre, Giza, Egypt.

\section{Acknowledgements}

The authors express sincere appreciation to Prof. Adel Bakeer Kholoussy, Professor of Pathology, Faculty of Veterinary Medicine, Cairo University, for his kind cooperation in conducting histological examination in this study.

\section{References}

Abd El Moety HA, El Sharkawy RM, Hussein NAE (2013). Lipocalin: A novel diagnostic marker for hepatocellular carcinoma in chronic liver disease patients in Egypt. Int $J$ Clin Med, 4, 440-50.

Abd-Rabou AA, Ahmed HH (2017). CS-PEG decorated PLGA nano-prototype for delivery of bioactive 4 compounds: A novel approach for induction of apoptosis in 5 HepG2 cell line. Adv Med Sci, 62, 357-67.

Aglan HA, Ahmed HH, El-Toumy SA, Mahmoud NS (2017). Gallic acid against hepatocellular carcinoma: An integrated scheme of the potential mechanisms of action from in vivo study. Tumor Biol, 39, 1-10.

Alves ACS, Mainardes RM, Khalil NM(2016). Nanoencapsulation of gallic acid and evaluation of its cytotoxicity and antioxidant activity. Mater Sci Eng C, 60, 126-34.

Asimakopoulou A, Weiskirchen S, Weiskirchen R (2016). Lipocalin 2 ( $(\mathrm{cn} 2)$ expression in hepatic malfunction and therapy. Front Physiol, 7, 430.
Ban JY, Nguyen HTT, Lee HJ, et al (2008). Neuroprotective properties of gallic acid from Sanguisorbae radix on amyloid $\beta$ protein (25-35)-induced toxicity in cultured rat cortical neurons. Biol Pharm Bull, 31, 149-53.

Bhattacharyya S, Ahammed SKM, Saha BP, Mukherjee PK (2013). The gallic acid-phospholipid complex improved the antioxidant potential of Gallic acid by enhancing its bioavailability. AAPS Pharm Sci Tech, 14, 1025-33.

Borkham-Kamphorst E, van de Leur E, Zimmermann HW,et al (2013). Protective effects of lipocalin-2 (LCN2) in acute liver injury suggest a novel function in liver homeostasis. Biochim Biophys Acta, 1832, 660-73.

Carleton H (1976). Carleton's Histological technique, 4th ed., University press: Oxford London, New York, Toronto. pp, 55-6.

Chen CY, Chen KC, Yang TY, Liu HC, Hsu SL (2013). Gallic Acid induces a reactive oxygen species-provoked c-Jun NH2-terminal kinase-dependent apoptosis in lung fibroblasts. Evid Based Complement Alternat Med, 2013, 613950.

Chen YJ, Chang LS (2012). Gallic acid downregulates matrixmetalloproteinase-2 (MMP-2) andMMP-9 in human leukemia cells with expressed Bcr/Abl. Mol Nutr Food Res, 56, 1398-412.

Chia YC, Rajbanshi R, Calhoun C, Chiu RH (2010). Anti-neoplastic effects of gallic acid, a major component of Toonasinensis leaf extract, on oral squamous carcinoma cells. Molecules, 15, 8377-89.

Darwish HA, El-Boghdady NA (2011). Possible involvement of oxidative stress in diethylnitrosamine induced hepatocarcinogenesis, chemopreventive effect of curcumin. $J$ Food Biochem, 37, 353-61.

Donelli MG, Zucchetti M, Munzone E, D’Incalci M, Crosignani A (1998). Pharmacokinetics of anticancer agents in patients with impaired liver function. Eur J Cancer, 34, 33-46.

Feng WY (2006). Metabolism of green tea catechins: an overview. Curr Drug Metab, 7, 755-809.

Ferruzzi MG, Lobo JK, Janle EM, et al (2009). Bioavailability of gallic acid and catechins from grape seed polyphenol extract is improved by repeated dosing in rats: implications for treatment in Alzheimer's disease. J Alzheimer Dis, 18, $113-24$.

Giodini L, Re FL, Campagnol D, et al (2017). Nanocarriers in cancer clinical practice: a pharmacokinetic issue. Nanomedicine, 13, 583-99.

Gonthier MP, Cheynier V, Donovan JL, et al (2003), Microbial aromatic acid metabolites formed in the gut account for a major fraction of the polyphenols excreted in urine of rats fed red wine polyphenols. J Nutr, 133, 461-7.

Heidarian E, Keloushadi M, Ghatreh-Samani K, Valipour P (2016). The reduction of IL-6 gene expression, pAKT, pERK1/2, pSTAT3 signaling pathways and invasion activity by gallic acid in prostate cancer PC 3 cells. Biomed Pharmacother, 84, 264-9.

Ho HH, Chang CS, Ho WC, et al (2013). Gallic acid inhibits gastric cancer cells metastasis and invasive growth via increased expression of Rho B, downregulation of AKT/ small GTPase signals and inhibition of NF-Kb activity. Toxicol Appl Pharmacol, 266, 76-85.

Hsiang CY, Hseu YC, Chang YC, et al (2013). Toonasinensis and its major bioactive compound gallic acid inhibit LPS-induced inflammation in nuclear factor- $\mathrm{Kb}$ transgenic mice as evaluated by in vivo bioluminescence imaging. Food Chem, 136, 426-34.

Jagan S, Ramakrishnan G, Anandakumar P, Kamaraj S, Devaki $\mathrm{T}$ (2008). Antiproliferative potential of gallic acid against diethylnitrosoamine-induced rat hepatocellular carcinoma. 
Mol Cell Biochem, 319, 51-9.

Karimov KHY, Inoyatova FKH, Mukhamedova MT (2003). Changes in some indices of the synthesis of nitric oxide during the early stages of hepatocarcinogenesis. Exp Toxicol Pathol, 55, 17-9.

Kee HJ, Cho SN, Kim GR, et al (2016). Gallic acid inhibits vascular calcification through the blockade of BMP2-Smad1/5/8 signaling pathway. Vascul Pharmacol, 63, 71-8.

Kim DO, Lee KW, Lee HJ, Lee CY (2002). Vitamin C equivalent antioxidant capacity of phenolic phytochemicals. J Agric Food Chem, 50, 3713-17.

Konishi Y, Hitomi Y, Yoshioka E (2004). Intestinal absorption of p-coumaric and gallic acids in rats after oral administration. J Agric Food Chem, 52, 2527-32.

Konishi Y, Zhao Z, Shimizu M (2006). Phenolic acids are absorbed from the rat stomach with different absorption rates. J Agric Food Chem, 54, 7539-43.

Kumar N, Biswas S, Mathew AE, et al (2016). Pro-apoptotic and cytotoxic effects of enriched fraction of Elytrantheparasitica (L.) Danser against HepG2 Hepatocellular carcinoma. BMC Complement Altern Med, 16, 420.

Lebrin F, Deckersm M, Bertolino P, Ten Dijke P (2005). TGF-h receptor functions in the endothelium. Cardiovasc Res, $\mathbf{6 5}$, 599-608.

Liang XJ, Chen C, Zhao Y, Wang PC (2010). Circumventing tumor resistance to chemotherapy by nanotechnology. Methods Mol Biol, 596, 467-88.

Liao CC, Chen SC, Huang HP, Wang CJ (2017). Gallic acid inhibits bladder cancer cell proliferation and migration via regulating fatty acid synthase (FAS) Q4. J food drug Analysis, in press.

Mard SA, Mojadami S, Farbood Y, GharibNaseri MK (2015). The anti-inflammatory and anti-apoptotic effects of gallic acid against mucosal inflammation- and erosions-induced by gastric ischemia-reperfusion in rats. Vet Res Forum, 6, 305-11.

Mohamed RA, Maghraby HM, Abd El Salam EM, et al (2015). Evaluation of serum endoglin as noninvasive marker in hepatocellular carcinoma. Egyptian Soc Intern Med, 27, 15-20.

Mansouri MT, FarboodY, Sameri MJ, et al (2013). Neuroprotective effects of oral gallic acid against oxidative stress induced by 6- hydroxydopamine in rats. Food Chem, 138, 1028-33.

Nabavi SF, Habtemariam S, Jafari M, Sureda A, Nabavi SM (2012). Protective role of gallic acid on sodium fluoride induced oxidative stress in rat brain. Bull Environ Contam Toxicol, 89, 73-7.

Nguyen TB, Roncalli M, Di Tommaso L, Kakar S (2016). Combined use of heat-shock protein 70 and glutamine synthetase is useful in the distinction of typical hepatocellular adenoma from atypical hepatocellular neoplasms and well-differentiated hepatocellular carcinoma. Mod Pathol, 29, 283-92.

Parveen S, Sahoo SK (2011). Long circulating chitosan/PEG blended PLGA nanoparticle for tumor drug delivery. Eur $J$ Pharmacol, 670, 372-83.

Perazzoli MR, Perondi CK, Baratto CM, et al (2017).Gallic acid and dodecyl gallate prevents carbon tetrachloride-induced acute and chronic hepatotoxicity by enhancing hepatic antioxidant status and increasing p53 expression. Biol Pharm Bull, 40, 425-34.

Pirmohamed M (2015). Prescribing in liver disease. Medicine, 43, 577-80

Priscilla DH, Prince PSM (2009). Cardioprotective effect of gallic acid on cardiac troponin-T, cardiac marker enzymes, lipid peroxidation products and antioxidants in experimentally induced myocardial infarction in wistar rats.
Chem Biol Interact, 179, 118-24.

Reddy TC, Reddy DB, Aparna A, et al (2012). Anti-leukemic effects of gallic acid on human leukemia K562 cells: downregulation of COX-2, inhibition of BCR/ABL kinase and NF-Kb inactivation. Toxicol In Vitro, 26, 396-405.

Rejiya CS, Cibin TR, Abraham A (2009). Leaves of Cassia tora as a novel cancer therapeutic--an in vitro study. Toxicol In Vitro, 23, 1034-8.

Sadeeshkumar V, Duraikannu A, Ravichandran S, et al (2016). Protective effects of dieckol on N-nitrosodiethylamine induced hepatocarcinogenesis in rats. Biomed Pharmacother, 2016, 1810-19.

Samarakoon SR, Shanmuganathan C, Ediriweera MK, et al (2017). Anti-hepatocarcinogenic and anti-oxidant effects of Mangrove Plant Scyphiphorahydrophyllacea. Pharmacogn Mag, 13, 76-83.

Santos NP, Colaço AA, Oliveira PA (2017).Animal models as a tool in hepatocellular carcinoma research: A Review. Tumor Biol, 39, 1-20.

Shahrzad S, Bitsch I (1998). Determination of gallic acid and its metabolites in human plasma and urine by high-performance liquid chromatography. J Chromatogr B, 705, 87-95.

Sobeh M, ElHawary E, Peixoto H, et al (2016). Identification of phenolic secondary metabolites from SchotiabrachypetalaSond.(Fabaceae) and demonstration of their antioxidant activities in Caenorhabditiselegans. Peer J, 4, e2404.

Sun G, Zhang S, Xie Y, Zhang Z, Zhao W (2016). Gallic acid as a selective anticancer agent that induces apoptosis in SMMC-7721 human hepatocellular carcinoma cells. Oncol Lett, 11, 150-8.

Sun Z, Zhao L, Zuo L, et al (2014). A UHPLC-MS/MS method for simultaneous determination of six flavenoids, gallic acid and 5,8-dihydroxy-1, 4-naphtho quinone in rat plasma and its application to a pharmacokinetic study of cortex Julandis Mandshuricae extract. J Chromatog B, 958, 55-62.

Sur S, Pal D, Mandal S, Roy A, Panda CK (2016). Tea polyphenols epigallocatechingallete and theaflavin restrict mouse liver carcinogenesis through modulation of self-renewal Wnt and hedgehog pathways. J NutrBiochem, 27, 32-42.

Tang MF, Lei L, Guo SR, Huang WL (2010). Recent progress in nanotechnology for cancer therapy. Chin J Cancer, 29, 775-80.

Teama S, Fawzy A, Teama S, et al (2016). Increased serum endoglin and transforming growth factor $\beta 1$ mRNA expression and risk of hepatocellular carcinoma in cirrhotic Egyptian patients. Asian Pac J Cancer Prev, 17, 2429-34.

Umadevi S, Gopi V, Simna SP, et al (2012). Studies on the cardio protective role of gallic acid against AGE-induced cell proliferation and oxidative stress in H9C2 (2-1) cells. Cardiovasc Toxicol, 12, 304-11.

Vaidyanathan JB, Walle T (2001). Transport and metabolism of the tea flavonoid (-)-epicatechin by the human intestinal cell line Caco-2. Pharm Res, 18, 1420-5.

Vaidyanathan JB, Walle T (2003). Cellular uptake and efflux of the tea flavonoid (-) epicatechin-3-gallate in the human intestinal cell line Caco-2. J Pharmacol Exp Ther, 307, $745-52$.

Verma S, Singh A, Mishra A (2013). Gallic acid: Molecular rival of cancer. Environ Toxicol Pharmacol, 35, 473-85.

Wang C, Zhang Y, Guo K, et al (2016). Heat shock proteins in hepatocellular carcinoma: Molecular mechanism and therapeutic potential. Int J Cancer, 138, 1824-34.

Yoon CH, Chung SJ, Lee SW, et al (2013). Gallic acid, a natural polyphenolic acid, induces apoptosis and inhibits proinflammatory gene expressions in rheumatoid arthritis fibroblast-like synoviocytes. Joint Bone Spine, 80, 274-9. 
Hanaa H Ahmed et al

Ziada DH, El Sadany S, Soliman H, et al (2016). Prevalence of hepatocellular carcinoma in chronic hepatitis $\mathrm{C}$ patients in Mid Delta, Egypt: A single center study. J Egypt Natl Canc Inst, 28, 257-62.

\section{(c) (i) (8)}

This work is licensed under a Creative Commons AttributionNon Commercial 4.0 International License. 